\title{
Projector quantum Monte Carlo with matrix product states
}

\author{
Sebastian Wouters, ${ }^{1, \text { f }}$ Brecht Verstichel, ${ }^{2}$ Dimitri Van Neck, ${ }^{1}$ and Garnet Kin-Lic Chan ${ }^{2, \dagger}$ \\ ${ }^{1}$ Center for Molecular Modelling, Ghent University, Technologiepark 903, 9052 Zwijnaarde, Belgium \\ ${ }^{2}$ Department of Chemistry, Princeton University, Princeton, New Jersey 08544, USA
}

\begin{abstract}
We marry tensor network states (TNS) and projector quantum Monte Carlo (PMC) to overcome the high computational scaling of TNS and the sign problem of PMC. Using TNS as trial wavefunctions provides a route to systematically improve the sign structure and to eliminate the bias in fixed-node and constrained-path PMC. As a specific example, we describe phaseless auxiliary-field quantum Monte Carlo with matrix product states (MPS-AFQMC). MPS-AFQMC improves significantly on the DMRG ground-state energy. For the $J_{1}-J_{2}$ model on two-dimensional square lattices, we observe with MPS-AFQMC an order of magnitude reduction in the error for all couplings, compared to DMRG. The improvement is independent of walker bond dimension, and we therefore use bond dimension one for the walkers. The computational cost of MPS-AFQMC is then quadratic in the bond dimension of the trial wavefunction, which is lower than the cubic scaling of DMRG. The error due to the constrained-path bias is proportional to the variational error of the trial wavefunction. We show that for the $J_{1}-J_{2}$ model on two-dimensional square lattices, a linear extrapolation of the MPS-AFQMC energy with the discarded weight from the DMRG calculation allows to remove the constrained-path bias. Extensions to other tensor networks are briefly discussed.
\end{abstract}

PACS numbers: 71.27.+a, 02.70.Ss, 75.10.Jm

\section{INTRODUCTION}

Tensor network states (TNS) and projector quantum Monte Carlo (PMC) are numerically exact methods for strongly correlated quantum states ${ }^{112}$ TNS provide compact parametrizations of quantum states in terms of local tensors, and become exact with increasing bond dimension $D$ [2]13 17 Matrix product states (MPS), the basis of the density matrix renormalization group (DMRG) $\stackrel{118 \mid 19}{1}$ are a widely successful example in oneand quasi-two-dimensional simulations. Although TNS provide an unbiased description of quantum states, they exhibit high computational scaling with $D$. For example, variational projected entangled pair states (PEPS) on a finite square lattice exhibit $\mathcal{O}\left(\chi^{2} D^{8}\right)$ scaling, with $\chi \geq D^{2}$ the virtual dimension trunctation in the approximate contraction, $2[3 / 13] 15$ which limits practical applicability.

PMC encompasses multiple methods with the common feature that the ground state $\left|\Psi_{*}\right\rangle$ is obtained by stochastically applying a projector, such as $\hat{K}=e^{-\delta \tau \hat{H}}$, to an ensemble of walkers $\sum_{k}\left|\phi_{k}\right\rangle{ }^{4 / 6|12| 20|22|}$ After sufficient applications, this ensemble stochastically represents the ground state $\left|\Psi_{*}\right\rangle$. For fermionic and frustrated systems, the walkers in PMC tend to represent both $\pm\left|\Psi_{*}\right\rangle$, leading to a vanishing signal-to-noise ratio for expectation values, the so-called sign problem. $\frac{10 \mid 23}{1}$ One way to approach fermionic and frustrated systems is to use fixed-node (FN) or constrained-path (CP) approximations, which eliminate the sign problem by constraining walkers to a fixed phase relative to an approximate trial

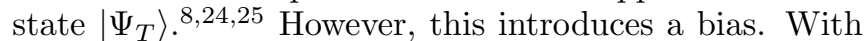
an improved description of the nodal structure in FN approximations, or the nodal plane in $\mathrm{CP}$ approximations, the bias becomes smaller.1026 34 The bias can often not be easily removed as common choices of trial states, such as Jastrow-Slaters, ${ }^{10129}$ cannot be easily improved ${ }^{35}$

Here, we present a marriage of TNS and PMC that combines their strengths and removes their respective weaknesses. Specifically, we combine MPS with phaseless auxiliary field quantum Monte Carlo (AFQMC) $\stackrel{819}{8}$ yielding MPS-AFQMC, although the ideas extend equally well to other combinations of TNS and PMC. MPS-AFQMC uses MPS as the trial state $\left|\Psi_{T}\right\rangle$ as well as to represent the walkers. This allows us to systematically remove the $\mathrm{CP}$ bias and improve the sign structure by increasing the trial bond dimension $D_{T}$, eliminating the main drawback of CP-PMC.

For the $J_{1}-J_{2}$ model on two-dimensional square lattices, we observe with MPS-AFQMC an order of magnitude reduction in the error for all couplings, compared to DMRG. The improvement is independent of walker bond dimension, and we therefore use bond dimension $D_{W}=1$ for the walkers. This leads to an $\mathcal{O}\left(D_{T}^{2}\right)$ computational scaling of MPS-AFQMC, which is lower than the $\mathcal{O}\left(D_{T}^{3}\right)$ scaling of the corresponding variational DMRG calculation. The high computational cost of TNS can therefore be addressed by a combined TNS-PMC approach. The error due to the $\mathrm{CP}$ bias is proportional to the variational error of the trial wavefunction. We show that for the $J_{1}-J_{2}$ model on two-dimensional square lattices, a linear extrapolation of the MPS-AFQMC energy with the discarded weight from the DMRG calculation allows to remove the constrained-path bias.

We note that in earlier work, TNS have been used with variational Monte Carlo $[461]$ However, this method can only stochastically reproduce the variational TNS energy of the ansatz under consideration, while PMC methods allow to improve on the variational ansatz. We mention also valence-bond basis projector Monte Carlo methods, which similarly use walkers in a complicated valencebond basis, but which so far have only been formulated 
for sign-free problems $\stackrel{42}{42}$

\section{PROJECTOR MONTE CARLO}

We begin with a brief overview of PMC methods before proceeding to MPS-AFQMC. PMC, encompassing lattice and real-space diffusion Monte Carlo (DMC), Green function Monte Carlo (GFMC), and auxiliary field quantum Monte Carlo (AFQMC), involves a choice of projector, walker basis, and $\mathrm{FN}$ or $\mathrm{CP}$ approximation.

Several PMC methods, including AFQMC, use the imaginary time propagator

$$
\hat{K}=e^{-\delta \tau \hat{H}} .
$$

The ground-state is obtained by

$$
\left|\Psi_{*}\right\rangle=\lim _{n \rightarrow \infty}(\hat{K})^{n}\left|\Psi^{(0)}\right\rangle, \quad \text { for }\left\langle\Psi_{*} \mid \Psi^{(0)}\right\rangle \neq 0 .
$$

Each application of $\hat{K}$ is denoted a time step. $\left|\Psi^{(n)}\right\rangle$, obtained after $n$ time steps, is represented by an ensemble of walkers

$$
\left|\Psi^{(n)}\right\rangle=\sum_{k}\left|\phi_{k}^{(n)}\right\rangle .
$$

Observables are obtained as averages over the ensemble; for example the (mixed) estimator for the energy is 43

$$
E_{T}^{(n)}=\sum_{k}\left\langle\Psi_{T}|\hat{H}| \phi_{k}^{(n)}\right\rangle / \sum_{k}\left\langle\Psi_{T} \mid \phi_{k}^{(n)}\right\rangle .
$$

Common choices of walkers include real-space coordinates, 10144 product spin states, 11 and Slater determinants (SDs), $\frac{89145}{2}$ and we will later introduce MPS walkers. To apply $\hat{K}$ to the walkers, we first express it as a summation (integral) over a probability distribution function (PDF) $P(\mathbf{x})$ and operators $\hat{B}(\mathbf{x})$

$$
\hat{K}=\sum_{\mathbf{x}} P(\mathbf{x}) \hat{B}(\mathbf{x}),
$$

where the choices of $P(\mathbf{x})$ and $\hat{B}(\mathbf{x})$ further differentiate the flavours of PMC. The only restriction in Eq. (5) is that $\hat{B}(\mathbf{x})$ maps a single walker to another walker of the same complexity: real-space coordinates should only change positions, SDs should remain SDs, or (in this work) the bond dimension of an MPS should not grow.

$\hat{K}$ is applied by sampling $\mathbf{x}$ with probability $P(\mathbf{x})$, and updating the walker:

$$
\left|\phi_{k}^{(n)}\right\rangle=\hat{B}(\mathbf{x})\left|\phi_{k}^{(n-1)}\right\rangle .
$$

A common way to improve statistics is to employ importance sampling with respect to a trial state $\left|\Psi_{T}\right\rangle$. Then the propagator is modified to

$$
\hat{K}_{\phi}=\sum_{\mathbf{x}} P(\mathbf{x}) \frac{\left\langle\Psi_{T}|\hat{B}(\mathbf{x})| \phi\right\rangle}{\left\langle\Psi_{T} \mid \phi\right\rangle} \hat{B}(\mathbf{x})=N_{\phi} \sum_{\mathbf{x}} \widetilde{P}_{\phi}(\mathbf{x}) \hat{B}(\mathbf{x}),
$$

where $N_{\phi}$ is the normalization to turn $\widetilde{P}_{\phi}(\mathbf{x})$ into a PDF. The importance sampling propagator biases moves towards regions where the overlap with $\left|\Psi_{T}\right\rangle$ is large. The ensemble now consists of weighted walkers

$$
\left|\Psi^{(n)}\right\rangle=\sum_{k} w_{k}^{(n)}\left|\phi_{k}^{(n)}\right\rangle .
$$

$N_{\phi}$ is accumulated into the weights

$$
w_{k}^{(n)}=N_{\phi_{k}^{(n-1)}} w_{k}^{(n-1)},
$$

which are controlled via branching. If the walker weights are smaller than 0.25 , or larger than $1.5,\left\lfloor w_{k}^{(n)}+u\right\rfloor$ copies of the walker are kept with weight 1 , with $u$ drawn from the uniform distribution on $[0,1[$. Note that this does not change the ensemble stochastically. In the importance sampling representation, the state and mixed estimator for the energy are

$$
\begin{aligned}
\left|\Psi^{(n)}\right\rangle & \propto \sum_{k} w_{k}^{(n)}\left|\phi_{k}^{(n)}\right\rangle /\left\langle\Psi_{T} \mid \phi_{k}^{(n)}\right\rangle, \\
E_{T}^{(n)} & =\frac{\sum_{k} w_{k}^{(n)}\left\langle\Psi_{T}|\hat{H}| \phi_{k}^{(n)}\right\rangle /\left\langle\Psi_{T} \mid \phi_{k}^{(n)}\right\rangle}{\sum_{k} w_{k}^{(n)}} .
\end{aligned}
$$

After sufficient time steps, the time-averaged ensemble stochastically represents $\left|\Psi_{*}\right\rangle$. Since only a limited number of walkers is used, the sampling bypasses the exponential complexity for the representation of a quantum state. The only issue is the sign (or phase) problem, i.e. $\pm\left|\Psi_{*}\right\rangle$ (or generally, $e^{i \theta}\left|\Psi_{*}\right\rangle$ with $\theta \in[0,2 \pi[$ ) are both fixed points of $\hat{K}$. Define the nodal plane $\mathcal{N}_{*} \cdot \frac{\sqrt{827 / 45}}{}$

$$
|\phi\rangle \in \mathcal{N}_{*} \Longleftrightarrow\left\langle\Psi_{*} \mid \phi\right\rangle=0 .
$$

If $|\phi\rangle$ can cross $\mathcal{N}_{*}$ to $-|\phi\rangle$ by successive application of the operators $\hat{B}(\mathbf{x})$ (which is the case for general fermion and frustrated spin propagators) then $\pm|\phi\rangle$ will occur with equal probability after infinite MC time. The signal representing $\left|\Psi_{*}\right\rangle$ then arises as a vanishing difference between populations of walkers representing $\pm\left|\Psi_{*}\right\rangle$, and estimators, such as the projected energy in Eq. (11), have large fluctuations from vanishing denominators $\left\langle\Psi_{T} \mid \phi_{k}^{(n)}\right\rangle$.

To recover a finite signal, we introduce the $\mathrm{CP}$ approximation. A trial wavefunction $\left|\Psi_{T}\right\rangle^{\sqrt{8|10| 45}}$ constrains the walker paths to one side of the nodal plane $\mathcal{N}_{T}$, by rejecting moves which change the sign of the overlap with $\left|\Psi_{T}\right\rangle$. This completely eliminates the sign problem. However, if $\left|\Psi_{T}\right\rangle$ is not exact and $\mathcal{N}_{*} \neq \mathcal{N}_{T}$, this introduces a systematic bias, which is the main drawback of CP-PMC. This is now the only remaining error in mixed estimators such as Eq. (11).

\section{MPS-AFQMC}

We now turn to MPS-AFQMC, the subject of this work. MPS (with open boundary conditions) are defined 
by

$$
|\phi\rangle=\sum_{\left\{s_{i}^{z} ; \alpha_{j}\right\}} A[1]_{\alpha_{1}}^{s_{1}^{z}} A[2]_{\alpha_{1} ; \alpha_{2}}^{s_{2}^{z}} . . A[L]_{\alpha_{L-1}}^{s_{L}^{z}}\left|s_{1}^{z} s_{2}^{z} . . s_{L}^{z}\right\rangle
$$

where the summation over each bond index $\alpha_{j}$ is truncated to $D$. By using MPS as the trial state (obtained in a prior variational DMRG calculation), we can increase the trial bond dimension $D_{T}$ to improve the CP approximation. This provides a systematic route to eliminate CP bias. We can also use MPS as walkers. To see this, consider the AFQMC decomposition for $\hat{K}$ with a Hubbard-Stratonovich (HS) transformation. ${ }^{[46}$ For concreteness, the spin Hamiltonian

$$
\hat{H}=\frac{1}{2} \sum_{i j} J_{i j} \hat{\vec{S}}_{i} \cdot \hat{\vec{S}}_{j}+\sum_{i} h_{i} \hat{S}_{i}^{z}
$$

is studied, with $J_{i j}=\sum_{k} V_{i k} \gamma_{k} V_{j k}$ symmetric. The (nonunique) HS transformation rewrites $e^{-\delta \tau \hat{H}}$, bilinear in the spin operators in the exponent, in terms of propagators with an exponent linear in the spin operators. Defining $\hat{v}_{k}^{w}=\sum_{i} \hat{S}_{i}^{w} V_{i k} \sqrt{-\gamma_{k}}$ with $w \in\{x, y, z\}$ we have:

$$
\begin{aligned}
\hat{H} & =\sum_{i} h_{i} \hat{S}_{i}^{z}-\sum_{w k} \frac{\left(\hat{v}_{k}^{w}\right)^{2}}{2}=\hat{H}_{1}-\sum_{w k} \frac{\left(\hat{v}_{k}^{w}\right)^{2}}{2}, \\
e^{-\delta \tau \hat{H}} & =\int d \mathbf{x} P(\mathbf{x}) \hat{B}(\mathbf{x})+\mathcal{O}\left(\delta \tau^{2}\right), \\
\hat{B}(\mathbf{x}) & =e^{-\delta \tau \hat{H}_{1} / 2} e^{\sqrt{\delta \tau} \mathbf{x} \cdot \hat{\mathbf{v}}} e^{-\delta \tau \hat{H}_{1} / 2} \\
P(\mathbf{x}) & =\frac{e^{-\mathbf{x}^{2} / 2}}{(2 \pi)^{3 L / 2}}
\end{aligned}
$$

with $\hat{\mathbf{v}}=\left(\hat{v}_{1}^{x}, \hat{v}_{1}^{y}, \hat{v}_{1}^{z}, \hat{v}_{2}^{x}, \ldots\right)$ and $L$ the number of lattice sites. Since $\hat{B}(\mathbf{x}) \equiv \prod_{i} \exp \left(\sum_{w} \alpha_{i}^{w} \hat{S}_{i}^{w}\right)$ is a product of single-site operators, applying $\hat{B}(\mathbf{x})$ to an MPS does not increase its bond dimension, allowing the use of MPS walkers. The walker bond dimension $D_{W}$ can be smaller than $D_{T}$ (if $D_{W}=1$, the walkers are product states) and this significantly reduces computational cost, as discussed below. We have also studied other operators $\hat{K}$ and other decompositions (5), ${ }^{47}$ but MPS-AFQMC was found to be the most promising variant.

The other aspects of MPS-AFQMC are formulated in precisely the same manner as standard phaseless AFQMC. For completeness, we briefly describe the phaseless CP approximation introduced by Zhang. ${ }^{9} \mathrm{Be}-$ cause $\hat{B}(\mathbf{x})$ in Eq. 17) can be complex, the AFQMC sign problem appears as a phase problem. The importance sampling propagator is implemented (up to $\left.\mathcal{O}\left(\delta \tau^{3 / 2}\right)\right)^{9}$ as a biased diffusion process

$$
\hat{K}_{\phi}=\int d \mathbf{x} P(\mathbf{x}) \hat{B}\left(\mathbf{x}-\mathbf{y}_{\phi}\right) N_{\phi}\left(\mathbf{x}, \mathbf{y}_{\phi}\right),
$$

where $\mathbf{y}_{\phi}$ applies a constant force drift, and importance sampling is achieved by choosing $\mathbf{y}_{\phi}=-\sqrt{\delta \tau} \frac{\left\langle\Psi_{T}|\hat{\mathbf{v}}| \phi\right\rangle}{\left\langle\Psi_{T} \mid \phi\right\rangle}$ which minimizes fluctuations in the normalization factor
$N_{\phi}(\mathbf{x}, \mathbf{y}) . \quad N_{\phi}\left(\mathbf{x}, \mathbf{y}_{\phi}\right)$ further takes the simple evocative form

$$
N_{\phi}\left(\mathbf{x}, \mathbf{y}_{\phi}\right) \approx \exp \left[-\delta \tau \frac{\left\langle\Psi_{T}|\hat{H}| \phi\right\rangle}{\left\langle\Psi_{T} \mid \phi\right\rangle}\right] \approx \exp \left[-\delta \tau E_{L}(\phi)\right](20)
$$

where $E_{L}(\phi)=\Re \frac{\left\langle\Psi_{T}|\hat{H}| \phi\right\rangle}{\left\langle\Psi_{T} \mid \phi\right\rangle}$ is the local energy. The phaseless approximation is imposed by forcing walkers to maintain a positive overlap with the trial state, modifying their weights by

$$
w_{k}^{(n)} \rightarrow w_{k}^{(n)} \max (0, \cos (\Delta \theta)),
$$

where $\Delta \theta$ is the phase of $\left\langle\Psi_{T} \mid \phi_{k}^{(n)}\right\rangle /\left\langle\Psi_{T} \mid \phi_{k}^{(n-1)}\right\rangle$. The quality of this nodal constraint depends on the quality of $\left|\Psi_{T}\right\rangle$, but as discussed above, by using MPS as $\left|\Psi_{T}\right\rangle$, the error can be completely removed by increasing $D_{T}$.

The main cost of MPS-AFQMC comes from computing the local energies $E_{L}\left(\phi_{k}\right)$ at each time step. If $\left|\Psi_{T}\right\rangle$ has bond dimension $D_{T}$ and the walkers bond dimension $D_{W}\left(D_{W}<D_{T}\right)$, then this only $\operatorname{costs} \mathcal{O}\left(D_{W} D_{T}^{2}\right)$, lower than the $\mathcal{O}\left(D_{T}^{3}\right)$ associated with expectation values in a variational DMRG calculation. For the $J_{1}-J_{2}$-model on 2D square lattices, both MPS-AFQMC and DMRG scale as $\mathcal{O}\left(L^{\frac{3}{2}}\right)$ in the system size, with $L$ the total number of lattice sites.

\section{SPIN- $\frac{1}{2} \quad J_{1}-J_{2}$ MODEL ON 2D SQUARE LATTICES}

To demonstrate the power of this new MPS-AFQMC approach, we now apply it to calculate the ground-state energies of the spin- $\frac{1}{2} J_{1}-J_{2}$ model on two-dimensional square lattices of sizes $4 \times 4$ and $6 \times 6$ with periodic boundary conditions $(\mathrm{PBC})$, and $8 \times 8$ and $10 \times 10$ with open boundary conditions (OBC). The $J_{1}-J_{2}$ model is defined by

$$
\hat{H}=J_{1} \sum_{\langle i j\rangle} \mathbf{S}_{i} \cdot \mathbf{S}_{j}+J_{2} \sum_{\langle\langle i j\rangle\rangle} \mathbf{S}_{i} \cdot \mathbf{S}_{j}
$$

in which $J_{1}$ is the coupling for nearest-neighbour spins, and $J_{2}$ the coupling for next-to-nearest neighbour spins. The $J_{1}-J_{2}$ model is of fundamental interest because it is one of the simplest models with frustration. For $J_{2}=0$, the model is the spin- $\frac{1}{2}$ Heisenberg model, whose groundstate is gapless and unfrustrated, and when $J_{2} / J_{1}=1$, the ground-state displays collinear striped magnetic order. In between, calculations show an intermediate phase in the region $0.4 \lesssim J_{2} / J_{1} \lesssim 0.62$ which appears gapped, and may be a $Z_{2}$ spin-liquid! 2148

The calculations begin with variationally optimizing an MPS (using a DMRG code) with trial dimension $D_{T}$. Subsequently, we use MPS-AFQMC to propagate an ensemble of MPS walkers with bond dimensions $D_{W}$. All calculations have been performed with a time step of $\delta \tau=0.01$, which was verified to yield a Trotter error within the range of the statistical error. We used sufficient time samples to obtain statistical error bars below 


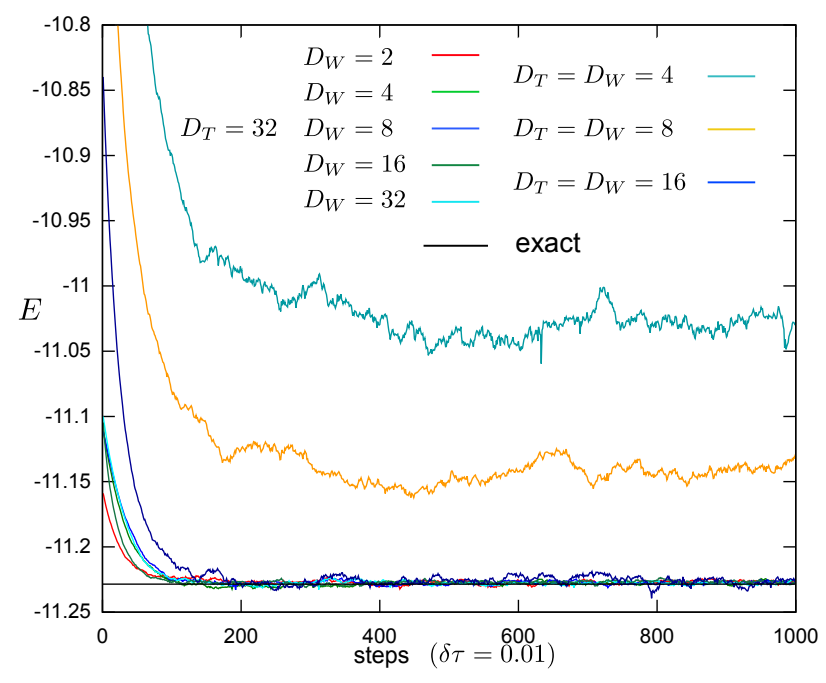

FIG. 1. Imaginary time evolution for a $4 \times 4 J_{1}-J_{2}$ model with $J_{2}=0$ using different values for the trial bond dimension $D_{T}$ and walker bond dimension $D_{W}$.

$0.01 \%$ in the energy. As a first check, we examine the dependence of the projected energy in Eq. (11) on the walker dimension $D_{W}$. Fig. 1 shows the projected energy as a function of imaginary time in the smallest $4 \times 4$ lattice with $J_{2} / J_{1}=0.0$, for a selection of $D_{W}$ and $D_{T}$. The zero-time energy ( $y$ intercept) is close to the DMRG energy, and the decrease of the curves shows the gain in accuracy using MPS-AFQMC. Interestingly, there appears to be no effect of walker bond dimension on either the final MPS-AFQMC energy or its statistical fluctuations. Thus the quality of the phaseless approximation depends only on $D_{T}$. We have therefore used walker bond dimension $D_{W}=1$ for the other calculations.

As seen from Fig. 1. MPS-AFQMC provides a substantial gain in accuracy over the initial DMRG energy. To see this more clearly, in Fig. 2 we show the converged MPS-AFQMC and DMRG energies for a variety of $D_{T}$ for the $4 \times 4$ lattice, with $J_{2} / J_{1}=0.6$. For this case, an MPS-AFQMC calculation with given $D_{T}$ reproduces the DMRG energy with a larger bond dimension of roughly $4 D_{T}$. Further, the convergence with $D_{T}$ is smooth for this system, resembling that of the DMRG energy itself. It is known that the DMRG energy can be extrapolated as a linear function of the discarded weight in the DMRG sweep algorithm. $\stackrel{47 / 49150}{ }$ Here, we obtain a similar linear dependence of the MPS-AFQMC energy with the DMRG discarded weight, as shown in the inset of Fig. 2. The CP error is therefore proportional to the variational error of the trial wavefunction. This allows us to perform high quality extrapolations to $D_{T}=\infty$ : in the case shown $\left(4 \times 4\right.$ lattice, $\left.J_{2} / J_{1}=0.6\right)$, we obtain $E\left(D_{T}=\infty\right)=-8.4133 \pm 0.0014$, in accordance with the exact result -8.4143 .

We next examine the accuracy of MPS-AFQMC across different regimes of frustration by studying the energy as $J_{2} / J_{1}$ is varied. Figs. 3,4 and 5 present the percentage

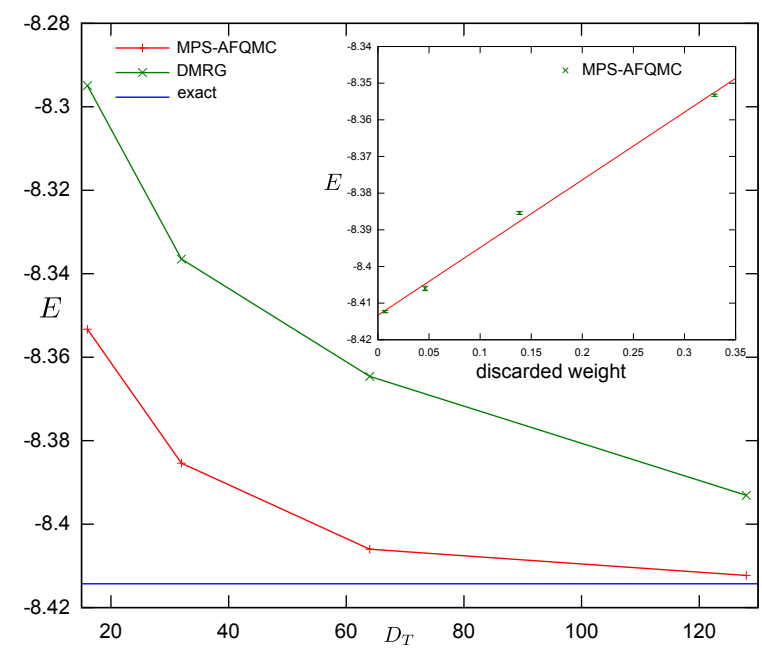

FIG. 2. Converged MPS-AFQMC and DMRG energies for different $D_{T}$ for a $4 \times 4 J_{1}-J_{2}$ model $(\mathrm{PBC})$ with $J_{2} / J_{1}=0.6$. Inset: extrapolation of the MPS-AFQMC energy with respect to discarded weight of the trial state.

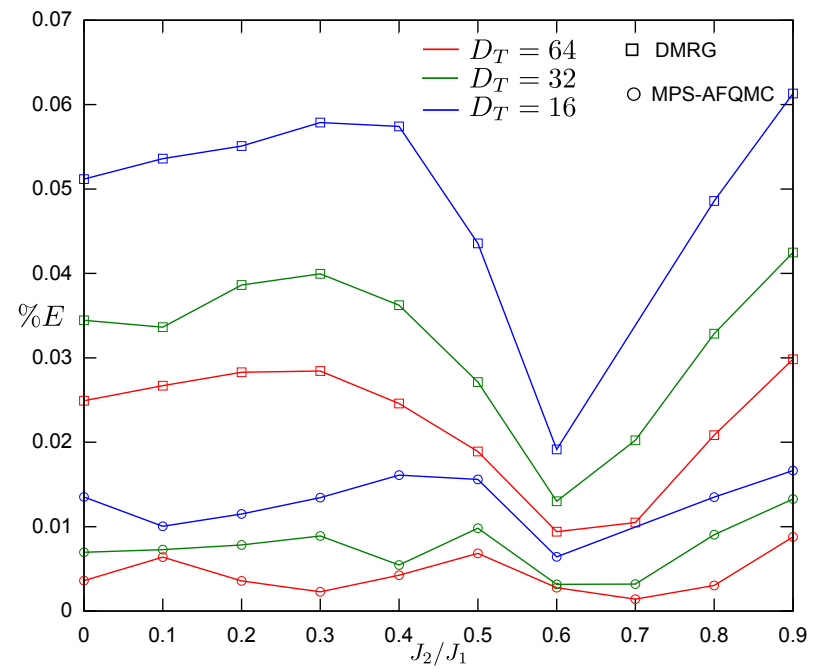

FIG. 3. Relative energy errors (from the exact result) for MPS-AFQMC and DMRG, as a function of $D_{T}$, for a $6 \times 6$ $J_{1}-J_{2}$ model (PBC) with $J_{2}$ ranging from 0 to $J_{1}$.

error in the MPS-AFQMC and DMRG energies relative to exact results for the $6 \times 6,8 \times 8$ and $10 \times 10$ lattices. The exact results were obtained from spin-adapted DMRG calculations with $D_{\mathrm{SU}(2)}=2000$ reduced renormalized basis states. Across all frustration regimes, the MPSAFQMC energy significantly improves on the DMRG energy, reducing the error by as much as $80 \%$, even in the highly frustrated regime. Overall, the MPS-AFQMC error tracks the modulations of the DMRG error as a function of $J_{2} / J_{1}$, with the energies being more accurate in the gapped intermediate coupling regime than in the gapless $J_{2} / J_{1}=0$ and $J_{2} / J_{1}=1$ limits.

The MPS-AFQMC calculations, which did not use symmetries, required comparable time to a toy varia- 


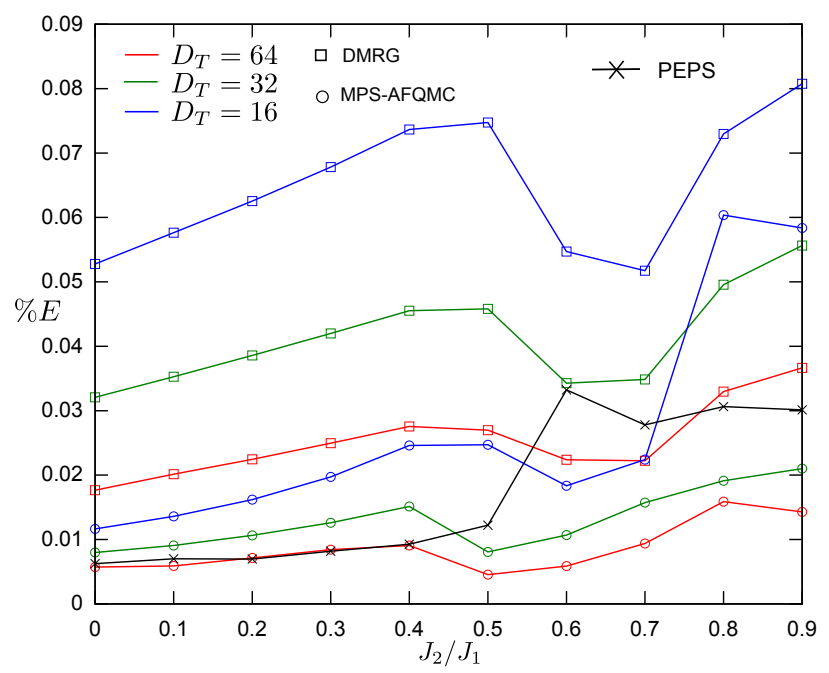

FIG. 4. Relative energy errors (from the exact result) for MPS-AFQMC and DMRG, as a function of $D_{T}$, for a $8 \times 8$ $J_{1}-J_{2}$ model (OBC) with $J_{2}$ ranging from 0 to $J_{1}$, compared to PEPS results from Ref. 38

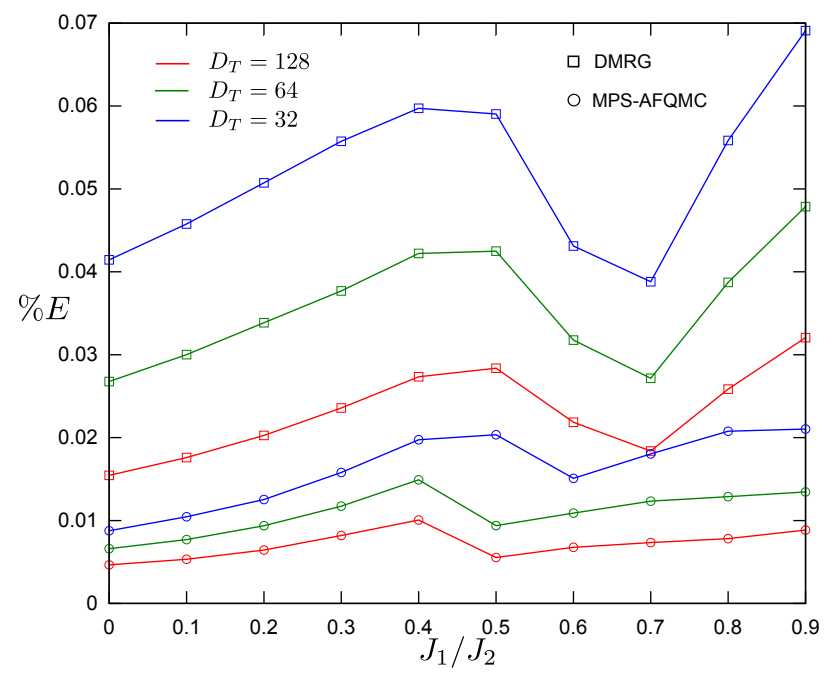

FIG. 5. Relative energy errors (from the exact result) for MPS-AFQMC and DMRG, as a function of $D_{T}$, for a $10 \times 10$ $J_{1}-J_{2}$ model (OBC) with $J_{2}$ ranging from 0 to $J_{1}$.

tional DMRG optimization of the trial state without symmetries for $D_{T} \approx 100$. In practice, we generated our trial states using our optimized spin-adapted DMRG code (with SU(2) symmetry). However, it is clear that for typical bond dimensions employed in DMRG $(D \approx 1000)$ and typical MPS-AFQMC parameters (100 walkers, 10000 time steps) PMC calculations will be highly competitive, if not faster, in timings, while achieving higher accuracy due to the effective bond dimension increase.

For higher-order TNS, the reduction in computational complexity due to the single-layer structure $\left(D_{W}=1\right)$ should be even more considerable. Further, PMC is highly parallel, in contrast to standard TNS optimiza- tion techniques.

When an MPS is used as a trial wavefunction for twodimensional lattices, $D_{T}$ has to increase exponentially with lattice width to maintain a constant accuracy. For large lattices, it is therefore better to resort to PEPS to parameterize the trial wavefunction.

\section{SUMMARY}

In conclusion, in this work we have described the marriage of tensor network states (TNS) and projector quantum Monte Carlo (PMC). The matrix product state auxiliary field quantum Monte Carlo (MPS-AFQMC) is a concrete realization of this marriage, which shows great promise. The use of an MPS trial wavefunction allows for a systematic removal of the $\mathrm{CP}$ error, which is the primary weakness of PMC methods in frustrated systems.

Further, the MPS-AFQMC method improves significantly on the variational DMRG ground-state energy, and does not depend on the bond dimension of the walkers. Product states $\left(D_{W}=1\right)$ can therefore be chosen as walkers. This leads to a computational cost which scales only quadratically in the bond dimension of the trial wavefunction. The increase in MPS-AFQMC accuracy over DMRG can also be interpreted as an effective bond dimension increase. We demonstrated these improvements for the spin-1/2 $J_{1}-J_{2}$ model on the square lattice. In addition, we observed a linear dependence of the MPS-AFQMC energy with the DMRG discarded weight. The $\mathrm{CP}$ error is therefore proportional to the variational error of the trial wavefunction.

While we have only presented energies in this work, other observables and correlation functions can be obtained in MPS-AFQMC following standard PMC techniques. ${ }^{10}$ In addition, while we have discussed spin systems in this work, fermionic MPS allow for a direct extension to fermions, including long-range Hamiltonians such as the Coulomb interaction in ab initio DMRG. 49 Finally, an important next step will be to extend these ideas to higher dimensional tensor networks, such as projected entangled pair states, 13 where the prohibitive computational scaling with bond dimension will be substantially reduced by PMC techniques, while providing greater accuracy than the corresponding variational calculation for the same bond dimension.

During the revision process, we discovered Refs. 54 and 55, Ref. 54 provides an earlier combination of DMRG with a different kind of MC, lattice DMC, within the fixed-node approximation, and contains similar ideas to the current work. Ref. 55, which appeared after our submission, also considered the combination of MPS and tree TNS with lattice DMC, with results that are comparable to using lattice AFQMC. 


\section{ACKNOWLEDGMENTS}

S.W. received a Ph.D. fellowship and B.V. a postdoctoral fellowship from the Research Foundation Flanders (FWO Vlaanderen). The work was supported by the US National Science Foundation through grants OCI1265278 and CHE-1265277.

* sebastianwouters@gmail.com

† gkc1000@gmail.com

1 U. Schollwöck, Ann. Phys. 326, 96 (2011)

2 R. Orus, arXiv 1306.2164 (2013).

3 F. Verstraete, V. Murg, and J. I. Cirac, Adv. Phys. 57, $143(2008)$

${ }^{4}$ M. H. Kalos, Phys. Rev. 128, 1791 (1962).

5 M. H. Kalos and P. A. Whitlock, Monte Carlo Methods, 2nd ed. (Wiley-VCH, 2008).

${ }^{6}$ R. Blankenbecler and R. L. Sugar, Phys. Rev. D 27, 1304 (1983)

R. Blankenbecler, D. J. Scalapino, and R. L. Sugar, Phys. Rev. D 24, 2278 (1981)

8 S. Zhang, J. Carlson, and J. E. Gubernatis, Phys. Rev. Lett. 74, 3652 (1995).

${ }^{9}$ S. Zhang and H. Krakauer, Phys. Rev. Lett. 90, 136401 (2003)

I0 W. M. C. Foulkes, L. Mitas, R. J. Needs, and G. Rajagopal, Rev. Mod. Phys. 73, 33 (2001).

11 N. Trivedi and D. M. Ceperley, Phys. Rev. B 41, 4552 (1990)

i2 G. H. Booth, A. J. W. Thom, and A. Alavi, J. Chem. Phys. 131, 054106 (2009)

${ }^{13}$ F. Verstraete and J. I. Cirac, arXiv condmat/0407066 (2004).

14 F. Verstraete and J. I. Cirac, Phys. Rev. A 70, 060302(R) (2004)

15 F. Verstraete, M. M. Wolf, D. Perez-Garcia, and J. I. Cirac, Phys. Rev. Lett. 96, 220601 (2006).

16 G. Vidal, Phys. Rev. Lett. 99, 220405 (2007).

17 G. Evenbly and G. Vidal, arXiv: 1210.1895 (2012).

18 S. R. White, Phys. Rev. Lett. 69, 2863 (1992).

19 S. R. White, Phys. Rev. B 48, 10345 (1993).

20 S. Sorella and L. Capriotti, Phys. Rev. B 61, 2599 (2000).

21 L. Capriotti and S. Sorella, Phys. Rev. Lett. 84, 3173 (2000)

${ }^{22}$ M. Boninsegni, Phys. Lett. A 216, 313 (1996).

23 S. Zhang, in Emergent Phenomena in Correlated Matter, Modeling and Simulation, Vol. 3, edited by E. Pavarini, E. Koch, and U. Schollwöck (Forschungszentrum Jülich, 2013) Chap. 15.

24 J. B. Anderson, Int. Rev. Phys. Chem. 14, 85 (1995)

${ }^{25}$ H. J. M. Van Bemmel, D. F. B. Ten Haaf, W. Van Saarloos, J. M. J. Van Leeuwen, and G. An, Phys. Rev. Lett. 72, 2442 (1994)

26 J. B. Anderson, J. Chem. Phys. 65, 4121 (1976)

27 D. M. Ceperley, J. Stat. Phys. 63, 1237 (1991).

28 W. A. Glauser, W. R. Brown, W. A. Lester, D. Bressanini, B. L. Hammond, and M. L. Koszykowski, J. Chem. Phys. 97, 9200 (1992)

29 M. Casula and S. Sorella, J. Chem. Phys. 119, 6500 (2003).
30 T. Mizusaki and M. Imada, Phys. Rev. B 69, 125110 (2004)

31 L. Mitas, Phys. Rev. Lett. 96, 240402 (2006)

32 C. J. Umrigar, J. Toulouse, C. Filippi, S. Sorella, and R. G. Hennig, Phys. Rev. Lett. 98, 110201 (2007).

${ }^{33}$ T. Aimi and M. Imada, J. Phys. Soc. Jpn. 76, 084709 (2007)

${ }^{34}$ H. Shi, C. A. Jiménez-Hoyos, R. Rodríguez-Guzmán, G. E. Scuseria, and S. Zhang, Phys. Rev. B 89, 125129 (2014)

${ }^{35}$ Y. Iqbal, F. Becca, S. Sorella, and D. Poilblanc, Phys. Rev. B 87, 060405(R) (2013)

उ० A. W. Sandvik and G. Vidal, Phys. Rev. Lett. 99, 220602 (2007)

3 H. J. Changlani, J. M. Kinder, C. J. Umrigar, and G. K.L. Chan, Phys. Rev. B 80, 245116 (2009).

38 F. Mezzacapo, N. Schuch, M. Boninsegni, and J. I. Cirac, New J. Phys. 11, 083026 (2009)

39 A. J. Ferris and G. Vidal, Phys. Rev. B 85, 165147 (2012)

40 E. Neuscamman, C. J. Umrigar, and G. K.-L. Chan, Phys. Rev. B 85, 045103 (2012).

${ }^{41}$ B. K. Clark, J. M. Kinder, E. Neuscamman, G. K.-L. Chan, and M. J. Lawler, Phys. Rev. Lett. 111, 187205 (2013).

${ }^{42}$ A. W. Sandvik and H. G. Evertz, Phys. Rev. B 82, 024407 (2010)

43 One of our referees pointed out the interesting connection of the mixed estimator in projector Monte Carlo to the concept of weak measurements in quantum computation.

44 R. C. Grimm and R. G. Storer, J. Comput. Phys. 7, 134 (1971)

45 S. Zhang, J. Carlson, and J. E. Gubernatis, Phys. Rev. B 55, 7464 (1997)

${ }^{40}$ R. L. Stratonovich, Soviet Physics-Doklady 2, 416 (1957).

$47 \mathrm{~S}$. Wouters, Accurate variational electronic structure calculations with the density matrix renormalization group, Ph.D. thesis, Ghent University (2014), arXiv 1405.1225 ISBN 9789461971944.

${ }^{48}$ H.-C. Jiang, H. Yao, and L. Balents, Phys. Rev. B 86, $024424(2012)$

${ }^{49}$ G. K.-L. Chan and M. Head-Gordon, J. Chem. Phys. 116, $4462(2002)$.

50 S. Wouters, W. Poelmans, P. W. Ayers, and D. Van Neck, Comput. Phys. Commun. 185, 1501 (2014)

51 S. R. White and R. L. Martin, J. Chem. Phys. 110, 4127 (1999)

52 O. Legeza, J. Röder, and B. A. Hess, Mol. Phys. 101, 2019 (2003)

${ }^{3}$ G. K.-L. Chan and S. Sharma, in Annu. Rev. Phys. Chem. Vol. 62, edited by S. R. Leone, P. S. Cremer, J. T. Groves, and M. A. Johnson (2011) pp. 465-481.

54 M. S. L. du Croo de Jongh, J. M. J. van Leeuwen, and W. van Saarloos, Phys. Rev. B 62, 14844 (2000) 
${ }^{55}$ B. K. Clark and H. J. Changlani, arXiv 1404.2296 (2014). 